\title{
Impact of several proinflammatory and cell degradation factors in patients with aortic valve stenosis
}

\author{
JURIS LURINS ${ }^{1}$, DACE LURINA ${ }^{2}$, SIMONS SVIRSKIS ${ }^{3}$, ZAIGA NORA-KRUKLE $^{3}$, PETERIS TRETJAKOVS ${ }^{4}$, \\ VITOLDS MACKEVICS ${ }^{1}$, AIVARS LEJNIEKS ${ }^{1}$, VENERANDO RAPISARDA $^{5}$ and VINCENZO BAYLON ${ }^{6}$ \\ ${ }^{1}$ Faculty of Medicine, Department of Internal Diseases, Riga Stradins University; \\ ${ }^{2}$ Latvian Maritime Medicine Centre; ${ }^{3}$ A. Kirchenstein Institute of Microbiology and Virology, Riga Stradins University; \\ ${ }^{4}$ Faculty of Medicine, Department of Human Physiology and Biochemistry, Riga Stradins University, Riga, LV 1007, Latvia; \\ ${ }^{5}$ Department of Clinical and Experimental Medicine, Occupational Medicine, \\ University Hospital 'Policlinico-Vittorio Emanuele', University of Catania, Catania I-95123, Italy; \\ ${ }^{6}$ Newton Lewis Institute Scientific Research-Life Science Park, San Gwann 3000, Malta
}

Received October 12, 2017; Accepted September 24, 2018

DOI: 10.3892/etm.2019.7254

\begin{abstract}
Aortic valve (AoV) stenosis is the third most common cardiovascular disease. The pathogenesis of AoV stenosis is associated with an inflammatory process where MMPs serve important roles. The aim of the present study was to determine the association between matrix metalloproteinases (MMPs), tissue inhibitors of metalloproteinases (TIMPs) and inflammatory factors, and AoV stenosis at various degrees of severity compared with the control. A total of 18 patients with mild, 19 with moderate and 15 with severe AoV stenosis were included in the present stud, and 50 individuals were enrolled in the control group. The severity of stenosis was determined by echocardiography. The expression levels of chemerin, fibroblast growth factor 21, MMP-1, -3, and -9, and TIMP-1 and -3 were analyzed by ELISA. Data were analyzed using GraphPad Prism7 software. The expression levels of MMP-1 was increased in patients with stenosis compared with the control group $(\mathrm{P}=0.0043)$. Distribution of the trimodal MMP-1 values was obtained in the stenosis group and monomodal in the control group. A total of $80 \%$ of patients in the stenosis group presented significantly increased
\end{abstract}

Correspondence to: Dr Juris Lurins, Faculty of Medicine, Department of Internal Diseases, Riga Stradins University, 16 Dzirciema Street, Riga, LV 1007, Latvia

E-mail: jl.cardio@gmail.com

Key words: aortic valve stenosis, matrix metalloproteinase-1, -3 and -9 , metalloproteinase inhibitor- 1 and -3 , chemerin, fibroblast growth factor 21, biomarker

Abbreviations: AoV, aortic valve; AVA, aortic valve area; MMPs, matrix metalloproteinases; FGF-21, fibroblast growth factor-21; TIMPs, tissue inhibitors of metalloproteinases; CRP, C-reactive protein expression levels of MMP-1 compared with the control group $(\mathrm{P}=0.0002)$. Expression of MMP-1 was significantly higher in all stenosis groups compared with the control. The highest expression level of MMP-1 appeared in patients with moderate stenosis $(\mathrm{P}<0.0001)$. There was no significant difference in the expression of MMP-3, MMP-9 and TIMP-1 in the aortic stenosis group, compared with the control group. A positive correlation between MMP-1 and MMP-9 expression levels was identified $(r=0.37 ; \mathrm{P}=0.017)$. The increase of MMP-1 was correlated with the increase of MMP-9, but not with the level of MMP-3. The expression levels of chemerin was significantly elevated in patients with stenosis compared with healthy patients. The highest expression levels of chemerin were determined in patients with mild $(\mathrm{P}=0.0001)$ and moderate $(\mathrm{P}=0.0007)$ stenosis and decreased with the grade of severity compared with the control group. The expression of FGF-21 was significantly different between the control and mild $(\mathrm{P}=0.013)$, moderate $(\mathrm{P}=0.015)$ and severe stenosis $(\mathrm{P}=0.003)$ groups. The expression levels of FGF-21 increased with the increase in severity grade, reaching the maximum for severe stenosis. The results of the present study indicated that the inflammatory process is predominantly occurring at the early, mild stage of stenosis and the most prominent extracellular matrix remodeling occurs in moderate stenosis (demonstrated by MMP-1 levels). In patients with severe stenosis, the levels of MMP-1 and chemerin (which are lower than in a case of mild or moderate stenosis) could indicate the development of calcinosis and the reduction in activity or inactivation of the inflammatory process.

\section{Introduction}

Acquired calcific aortic valve (AoV) stenosis is the most common valvular disease in the USA and Europe and is the second most frequent cause for cardiac surgery (1). The prevalence of aortic valve stenosis is $0.2 \%$ among adults aged $50-59$ years, $1.3 \%$ among patients aged $60-69$ years, and $\leq 9.8 \%$ among patients aged $80-89$ years (2). 
Overall, AoV stenosis is present in $2.6 \%$ of adults $>75$ years of age (3). The average time from diagnosis of aortic sclerosis to the development of moderate and severe AoV stenosis is 6 and 8 years, respectively (4).

Among symptomatic patients with medically treated moderate-to-severe aortic stenosis, mortality from the onset of symptoms is $25 \%$ at 1 year and $50 \%$ at 2 years (5). Symptoms of aortic stenosis usually develop gradually after an asymptomatic latent period of 10-20 years (6).

AoV stenosis has been previously considered to be the cause of aging and atherosclerosis; however, in recent years this view has changed. At present, AoV stenosis is considered to be an active process. Inflammatory factors, extracellular matrix remodeling and calcification processes serve important roles in the pathogenesis of AoV stenosis (7).

Calcific AoV stenosis is characterized by cell proliferation and development of remodeling processes in the extracellular matrix. Matrix metalloproteinases (MMPs) serve important roles in the remodeling processes, which leads to the degradation of the extracellular matrix (8-10).

MMPs are some of the most frequently studied extracellular matrix proteins in the pathogenesis of calcific AoV stenosis (11). MMPs are zinc-dependent endopeptidases (collagenases, gelatinases and stromelysins) that serve roles in both physiological and pathological remodeling of the extracellular matrix in a number of tissues and diseases (12). Tissue remodeling is affected by the local balance between MMPs and endogenous tissue inhibitors of metalloproteinases (TIMPs) $(13,14)$. The present study determined the expression levels of MMP-1, -3 and -9 in the blood serum and the corresponding TIMP-1 and -3 expression. These MMPs, in particular MMP-1 and MMP-9, have been described by numerous researchers who have investigated their possible association with stenosis $(12,13,15,16)$. However, mainly as a material using tissues, removed during the aortic valve replacement surgery, consequently, these studies cannot compare the changes between severity degrees of aortic valve stenosis. The number of histological materials included in these previous studies was frequently small and there were ethical restrictions associated with the establishment of appropriate control groups.

The current study aimed to clarify the factors that serve an active role in the development of AoV stenosis, and to determine their presence and significance by determining their serum levels in patients with aortic valve stenosis of different severity degrees in comparison with the control group. This method was used to estimate the sequence of processes occurring during the development of aortic valve stenosis.

C-reactive protein is known as a stable inflammatory factor and a marker of inflammation, and chemerin is a relatively new biomarker involved in various inflammatory processes. Chemerin has been previously demonstrated to be released by fibroblasts in the heart (17). FGF-21, that has been described as a factor with a broad spectrum of manifestations, may serve a cardioprotective role, and may contribute to the development of fibrosis and may be excreted by cardiac cells (18-21).

The aim of the present study was to demonstrate the involvement of inflammatory factors and MMPs in the development of aortic valve stenosis, their role in progression of the disease according to the AoV stenosis severity grades, and, therefore indirectly provide evidence of the possible sequence and mechanism of the development of AoV stenosis.

\section{Patients and methods}

Study population. The current study was conducted from January 2013 until the end of December 2016 at the Vidzeme Regional Hospital (Valmiera, Latvia), P. Stradins Clinical University Hospital (Riga, Latvia) and at the polyclinicZemgale Health Centre (Jelgava, Latvia). The study protocol was approved by the Riga Stradins University Ethics Committee on Research on Humans and the study protocol conforms to the Ethical Guidelines of the 1975 Declaration of Helsinki. A total of 102 patients were selected according to inclusion and exclusion criteria and divided in two core groups, an AoV stenosis group and a control group. The control group included patients without AoV stenosis, aged 55-80 years, which matched the average age of patients with AoV stenosis according to the Guidelines on the Management of Valvular Heart Disease 2012 of the European Society of Cardiology (22). Written informed consent was obtained from each patient enrolled in the current study. Patients were included in the control group according to echocardiographically confirmed results of a healthy aortic valve. Exclusion criteria in both groups included: Obesity, systemic connective tissue diseases, infectious diseases, oncological diseases, diabetes, thyroid dysfunction, moderate, severe and uncontrolled arterial hypertension, with acute coronary syndrome in anamnesis and coronary heart disease, left ventricular systolic dysfunction with reduced ejection fraction below $40 \%$, cerebral infarction, transient ischemic attack, echocardiographically confirmed cardiomyopathy and pathology of other valves. Exclusion criteria in the $\mathrm{AoV}$ stenosis group included congenital and rheumatic aortic valve stenosis.

Aortic valve stenosis assessment. All patients were examined echocardiographically and the data were obtained using GE VIVID 7 Dimension Cardiovascular Ultrasound system (GE Healthcare; GE Healthcare, Chicago, IL, USA) and Philips iE 33 Ultrasound Machine (Philips Healthcare, Amsterdam, The Netherlands). Each echocardiography examination was evaluated by 2 professionals. Patients with AoV stenosis were subdivided into three groups depending on the severity grade according to the echocardiography criteria, including aortic jet velocity $[\operatorname{Vmax}(\mathrm{m} / \mathrm{s})]$, mean pressure gradient $[\mathrm{PG}(\mathrm{mmHg})]$, aortic valve area [AVA $\left.\left(\mathrm{cm}^{2}\right)\right]$ and indexed AVA $\left(\mathrm{cm}^{2} / \mathrm{m}^{2}\right)$. The following groups were established: i) Severe (Vmax $>4 \mathrm{~m} / \mathrm{sec}$; $\mathrm{PG}>40 \mathrm{mmHg}$; AVA $<1.0 \mathrm{~cm}^{2}$; indexed AVA $<0.6$ ); ii) moderate (Vmax 3.0-4.0 m/sec; PG 20-40 mmHg; AVA 1.0-1.5 cm²; indexed AVA 0.60-0.85); and iii) mild (Vmax 2.5-2.9 m/sec; $\mathrm{PG}<20 \mathrm{mmHg}$; AVA $>1.5 \mathrm{~cm}^{2}$; indexed AVA $>0.85$; Table I).

Biochemical analysis. Venous blood samples were collected and serum was obtained in accordance with the serum preparation instructions (23). Serum samples were stored at $-80^{\circ} \mathrm{C}$ and were available for all 102 patients. The concentration of lipids and C-reactive protein were analyzed via standard methods at the laboratory of the Pauls Stradins Clinical University Hospital (Riga, Latvia). Total cholesterol was determined using the commercially available test [ADVIA ${ }^{\circledR}$ Chemistry Cholesterol_2 (CHOL_2); cat. no. 10376501], Siemens Healthineers, Erlangen, Germany], based on an enzymatic method that utilizes cholesterol esterase and cholesterol 
Table I. Baseline characteristics of the patients.

\begin{tabular}{|c|c|c|c|c|}
\hline Characteristic & $\begin{array}{l}\text { Control, } \\
n=50\end{array}$ & $\begin{array}{l}\text { AoV mild stenosis, } \\
\qquad \mathrm{n}=18\end{array}$ & $\begin{array}{l}\text { AoV moderate stenosis, } \\
\qquad \mathrm{n}=19\end{array}$ & $\begin{array}{c}\text { AoV severe stenosis, } \\
n=15\end{array}$ \\
\hline \multicolumn{5}{|l|}{$\operatorname{Sex}(\%)$} \\
\hline Male & $11(22.0)$ & $2(11.1)$ & $8(42.1)$ & $7(46.7)$ \\
\hline Female & $39(78.0)$ & $16(88.9)$ & $11(57.9)$ & $8(53.3)$ \\
\hline \multirow[t]{2}{*}{ Age } & $65.18(9.74)$ & $70.53(6.08)$ & $72.16(8.20)$ & $65.27(8.13)$ \\
\hline & & $\mathrm{P}=0.127$ & $\mathrm{P}=0.012$ & $P>0.999$ \\
\hline \multirow[t]{2}{*}{ BMI } & $26.04(4,31)$ & $27.39(3,10)$ & $25.81(4,58)$ & $27.40(3,18)$ \\
\hline & & $\mathrm{P}=0.399$ & $\mathrm{P}=0.682$ & $\mathrm{P}=0.869$ \\
\hline \multirow[t]{2}{*}{$\mathrm{LDL}, \mathrm{mmol} / \mathrm{l}$} & $3.28(1.18)$ & $3.05(0.97)$ & $2.59(0.92)$ & $3.10(1.12)$ \\
\hline & & $\mathrm{P}>0.999$ & $\mathrm{P}=0.057$ & $\mathrm{P}>0.999$ \\
\hline \multirow[t]{2}{*}{ Triglycerides, mmol/1 } & $1.47(0.71)$ & $1.64(0.84)$ & $1.11(0.56)$ & $1.27(0.57)$ \\
\hline & & $\mathrm{P}=0.406$ & $\mathrm{P}=0.178$ & $\mathrm{P}=0.406$ \\
\hline \multirow[t]{2}{*}{ Total cholesterol, mmol/1 } & $5.49(1.28)$ & $5.01(1.34)$ & $4.21(1.18)$ & $4.68(1.08)$ \\
\hline & & $\mathrm{P}=0.056$ & $\mathrm{P}=0.001$ & $\mathrm{P}=0.016$ \\
\hline \multirow[t]{2}{*}{ CRP, mg/l } & $0.95(0.50-2.55)$ & $3.00(1.5-3.70)$ & $1.75(0.37-2.97)$ & $1.2(0.7-5.00)$ \\
\hline & & $\mathrm{P}=0.016$ & $\mathrm{P}=0.37$ & $\mathrm{P}=0.17$ \\
\hline \multirow[t]{2}{*}{$\mathrm{SV}, \mathrm{ml}$} & $81.86(20.11)$ & $73.63(18.92)$ & $77.72(18.65)$ & $75.93(15.49)$ \\
\hline & & $\mathrm{P}=0.299$ & $\mathrm{P}=0.501$ & $\mathrm{P}=0.501$ \\
\hline \multirow[t]{2}{*}{$\mathrm{EF}, \%$} & $62.22(6.35)$ & $57.16(9.23)$ & $61.78(8.23)$ & $56.13(7.84)$ \\
\hline & & $\mathrm{P}=0.014$ & $\mathrm{P}=0.291$ & $\mathrm{P}=0.007$ \\
\hline
\end{tabular}

All data are presented as the mean ( \pm standard deviation), with the exception of CRP levels presented as the median and interquartile range. All P-values are vs. the control group. BMI, weight in kilograms divided by the square of the height in meters; LDL, low-density lipoprotein cholesterol; CRP is C-reactive protein; SV, stroke volume, measured by left ventricular outflow method; EF, ejection fraction, measured by Simpson's method.

oxidase conversion, followed by a Trinder endpoint (24). The cholesterol esters were hydrolyzed by cholesterol esterase to form cholesterol and free fatty acids. The cholesterol was then converted to cholest-4-en-3-one by cholesterol oxidase in the presence of oxygen to form hydrogen peroxide. A colored complex was then formed from hydrogen peroxide, 4-aminoantipyrine and phenol under the catalytic influence of peroxidase. The absorbance of the complex was measured as an endpoint reaction at 505/694 $\mathrm{nm}$. Triglycerides were determined using commercially the available test $\left[\right.$ ADVIA ${ }^{\circledR}$ Chemistry Triglycerides_2 (TRIG_2) Reagent; cat. no. REF 10335892]; Siemens Healthineers], based on the Fossati three-step enzymatic reaction with a Trinder endpoint. The single-reagent procedure quantitates the total triglycerides including the monoglycerides, diglycerides and free glycerol fractions. The triglycerides were converted to glycerol and free fatty acids by lipase. Glycerol was then converted to glycerol-3-phosphate by glycerol kinase, which was further converted with glycerol-3-phosphate-oxidase to form hydrogen peroxide. A colored complex was formed from hydrogen peroxide, 4-aminophenazone and 4-chlorophenol under the catalytic influence of peroxidase. The absorbance of the complex was measured as an endpoint reaction at 505/694 nm. High-density lipoprotein cholesterol and low-density lipoprotein cholesterol were determined using commercially available tests [ADVIA ${ }^{\circledR}$ Chemistry Direct HDL Cholesterol (D-HDL) Reagent; cat. no. 07511947 and ADVIA ${ }^{\circledR}$ Chemistry LDL Cholesterol
Direct (DLDL) Reagent; cat. no. 09796248 respectively]; Siemens Healthcare Diagnostics Inc., Tarrytown, USA], using the direct method. Direct high-density lipoprotein cholesterol method measured HDL cholesterol in serum without prior separation, based on procedures developed by Izawa et al (25). Cholesterol from non-HDL particles was released and eliminated in the first step of the reaction. Cholesterol in HDL particles was further released in the second step by detergent in Reagent 2 [component of the ADVIA ${ }^{\circledR}$ Chemistry Direct HDL Cholesterol (D-HDL) kit] and the HDL cholesterol was measured via a Trinder reaction. The low-density lipoprotein cholesterol direct method measured LDL cholesterol in serum. The first step of the reaction eliminated cholesterol associated with lipoproteins other than low-density lipoprotein. A selective surfactant [component of the ADVIA ${ }^{\circledR}$ Chemistry LDL Cholesterol Direct (DLDL) kit] released cholesterol preferentially from non-LDL particles. Hydrogen peroxide produced by cholesterol esterase and cholesterol oxidase in the first step was then eliminated by catalase. Another surfactant in Reagent 2 [component of the ADVIA ${ }^{\circledR}$ Chemistry LDL Cholesterol Direct (DLDL) kit] released cholesterol from the low density lipoprotein. Azide in R2 inhibited the catalase. Hydrogen peroxide generated by cholesterol esterase and cholesterol oxidase was then quantified using a Trinder endpoint. All methods were performed using the Siemens Advia 1800 analyzer (Siemens Healthcare Diagnostics Inc., Tarrytown, NY, USA) in accordance with the manufacturer's protocol. C-reactive protein was 
determined using the particle enhanced turbidimetric method. Human CRP was determined using commercially available test where CRP was agglutinated with latex particles coated with monoclonal anti-CRP antibodies (cat. no. CRPLX; Roche Diagnostics, Basel, Switzerland). The precipitate is determined turbidimetrically at $552 \mathrm{~nm}$. This was performed using the Roche Cobas Integra 400 Plus analyzer (Roche Diagnostics), according to the manufacturer's protocol. Analyses of MMP-1, MMP-3, MMP-9, TIMP-1, TIMP-3, chemerin and FGF-21 were performed at the biochemical laboratory of the Riga Stradins University (Riga, Latvia), using the following ELISA kits: Human MMP-1 (cat. no. EHMMP1; Pierce; Thermo Fisher Scientific, Inc., Waltham, MA, USA); human MMP-3 (cat. no. ELH-MMP3; RayBiotech, Inc., Norcross, GA, USA); human MMP-9 (cat. no. KHC3061; Invitrogen; Thermo Fisher Scientific, Inc.); human TIMP-1 (cat. no. ab100651; Abcam, Cambridge, UK); human TIMP-3 (cat. no. ab119608; Abcam); human Chemerin ELISA (cat. no. EZHCMRN-57K; Merck KGaA, Darmstadt, Germany) and human FGF-21 ELISA (cat. no. EZHFGF21-19K; Merck Millipore, USA). Results were detected using an Infinite 200 PRO multimode reader (Tecan Group, Ltd., Mannedorf, Switzerland) and Multiskan Ascent microplate reader (Thermo Labsystems, Helsinki, Finland). The procedure was performed in accordance with the ELISA kit manufacturer's protocol.

Statistical analysis. All the graphs, calculations, and statistical analyses were performed using GraphPad Prism software version 7.0 for Mac (GraphPad Software, Inc., La Jolla, CA, USA). Comparison of means between different groups was performed using one-way analysis of variance (ANOVA). Brown-Forsythe and Bartlett's tests were applied to determine whether the obtained data were normally distributed. In the case of unequal standard deviations, comparisons of medians between different groups were performed with non-parametric one-way ANOVA on ranks or Kruskal-Wallis test followed by post-hoc analysis. In all cases two-stage step-up method of Benjamin, Krieger and Yekutieli was used as post-hoc analysis (26). Baseline statistics are presented in Table I. All results for age, body mass index, low-density lipoprotein cholesterol, total cholesterol, triglycerides and ejection fraction (measured by Simpson's method), stroke volume (measured by left ventricular outflow method) (27) are presented as the mean \pm standard deviation. Results for high-sensitivity C-reactive protein are presented as the median and interquartile range. As the distribution of data was logarithmic, the geometric mean and geometric standard deviation of the data were used to represent the results. A two-tailed $\mathrm{P}<0.05$ was considered to indicate a statistically significant difference. The correlation analysis method was used to study the associations between quantitative variables. Depending on the data distribution, parametric (Pearson's) or non-parametric (Spearman's) correlation analyses were performed and corresponding characteristics $\left(r_{p}\right.$ or $r_{s}$ ) were used.

\section{Results}

Patient characteristics. Patient characteristics are presented in Table I. A total of 102 patients were included and divided into three AoV stenosis severity groups and a control group. A total of 18 patients presented mild, 19 moderate, and 15 severe AoV stenosis, and there were 50 patients without AoV stenosis in the control group. The aforementioned exclusion criteria allowed the selection of appropriate study groups. Although the number of patients in the subgroups was limited, the statistical analysis revealed significant $\mathrm{P}$-values in the range $\mathrm{P}<0.05-0.0001$.

The average age of patients in all of the aortic stenosis groups and the control group were similar, and the mean level of body mass index did not differ between the groups. Triglyceride and low-density lipoprotein cholesterol mean values did not differ significantly between the stenosis groups and the control group. The aortic stenosis patient groups were similar in respect to the mean values of ejection fraction, measured by Simpson's method and stroke volume, measured by left ventricular outflow method. However, ejection fraction in severe aortic stenosis group was significantly decreased $(\mathrm{P}=0.007)$ in comparison with the control group. The expression of $\mathrm{C}$-reactive protein was statistically significantly higher $(\mathrm{P}=0.016)$ among patients with mild stenosis compared with the control patients (Fig. 1).

Differences in chemerin expression levels between the patient groups. The mean chemerin expression level among all patients with AoV stenosis differed significantly $(\mathrm{P}<0.0001)$ compared with the control group (Fig. 2). A more detailed analysis of AoV stenosis subgroups, including mild, moderate and severe(Fig. 3), demonstrated that chemerin level was significantly elevated in all three AoV stenosis groups compared with the control patients and the most pronounced effect was observed in the mild AoV stenosis group $(\mathrm{P}=0.0001)$.

Chemerin level in the mild AoV stenosis group was also significantly higher compared with the severe AoV stenosis group $(\mathrm{P}<0.05)$, while no significant difference between moderate and severe AoV stenosis groups was observed. Elevated chemerin levels decreased with the increase of AoV stenosis severity, reaching the lowest level in patients with severe aortic stenosis (Fig. 3). This effect was confirmed by regression analysis, where a linear regression line with a negative slope was determined (Fig. 4).

Differences in FGF-21 expression levels between the patient groups. The serum expression levels of FGF-21 were significantly higher among patients AoV stenosis compared with the control group $(\mathrm{P}<0.0001$; Fig. 5). The most significant difference was observed in the severe stenosis group $(\mathrm{P}=0.003)$; however, significant differences were also observed in patients with moderate $(\mathrm{P}=0.015)$ and mild $(\mathrm{P}=0.013)$ stenosis groups compared with the control group (Fig. 6). The increase of FGF-21 serum level (depending on the degree of AoV stenosis), in difference to chemerin, gradually increased, exhibiting a linear tendency, which is presented in Fig. 7 as positive slope of regression. A positive association was observed between the expression of chemerin and FGF-21 in the control group $\left(r_{p}=0.34 ; P=0.019\right)$, and the aortic valve stenosis group $\left(r_{p}=0.35\right.$; $\mathrm{P}=0.012$ ) (Fig. 8A and B). An increase in the chemerin level was associated with an increase in FGF-21 concentration. A positive correlation between chemerin expression and serum levels of MMP-9 was also detected (Fig. 8C; $\mathrm{r}_{\mathrm{p}}=0.362 ; \mathrm{P}=0.008$ ); however, no association between chemerin and high-density lipoproteins (HDL) was identified. 


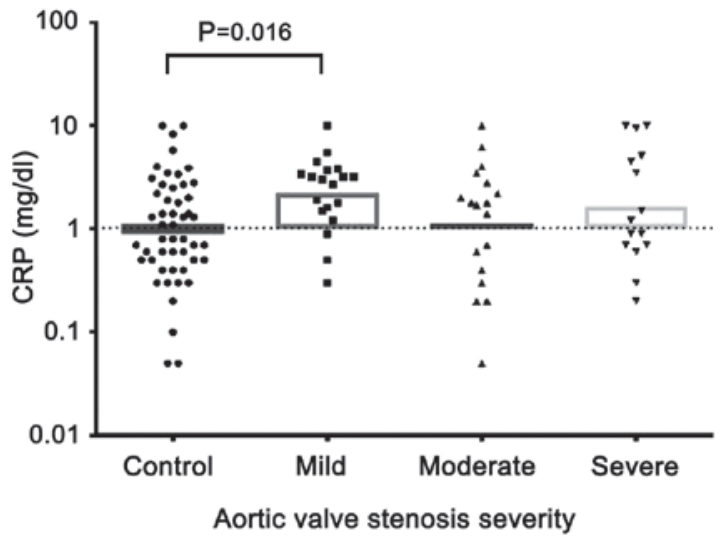

Figure 1. Serum CRP level in the control group and among patients with different degrees of aortic valve stenosis severity. CRP, C-reactive protein.

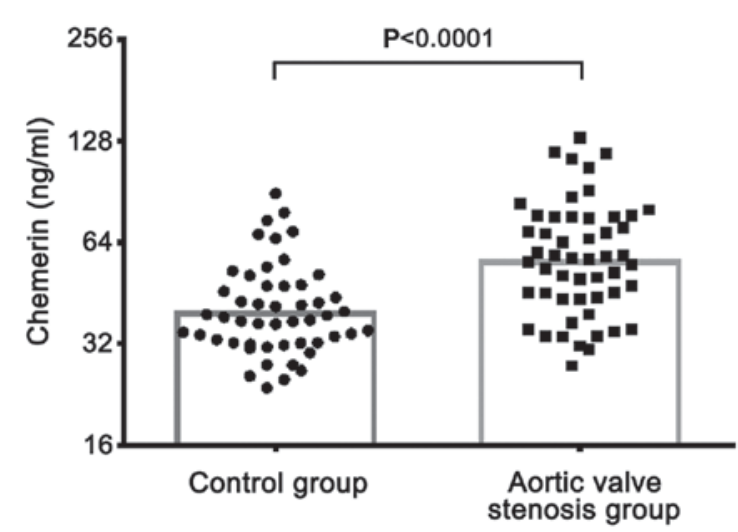

Figure 2. Serum chemerin level in the control and aortic valve stenosis stenosis groups.

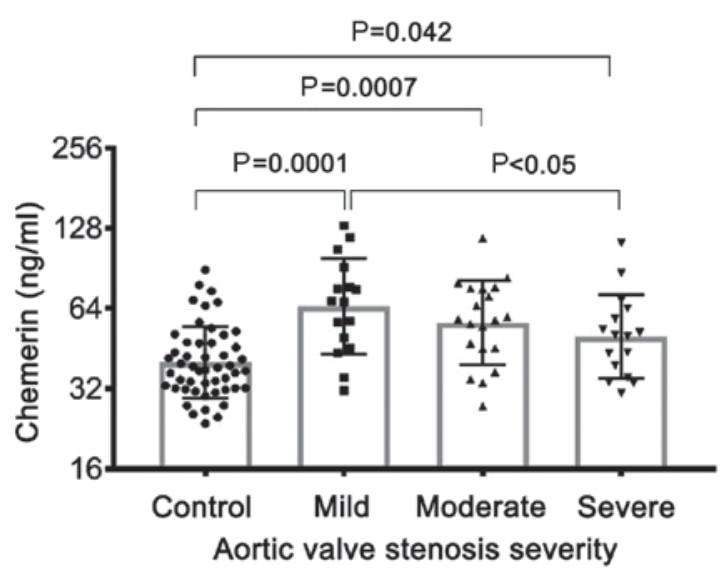

Figure 3. Serum Chemerin level in the control group and in AoV stenosis groups with different grades of severity.

Differences in the serum levels of MMPs in patients with different degrees of aortic valve stenosis. The expression of MMP-1 was also significantly increased in patients with AoV stenosis compared with the control group $(\mathrm{P}=0.0043)$. However, the results and their distribution were different from those obtained with chemerin and FGF-21. It was also demonstrated that patients with aortic valve stenosis exhibited a dual distribution of the response (Fig. 9A). Therefore, the values of MMP-1

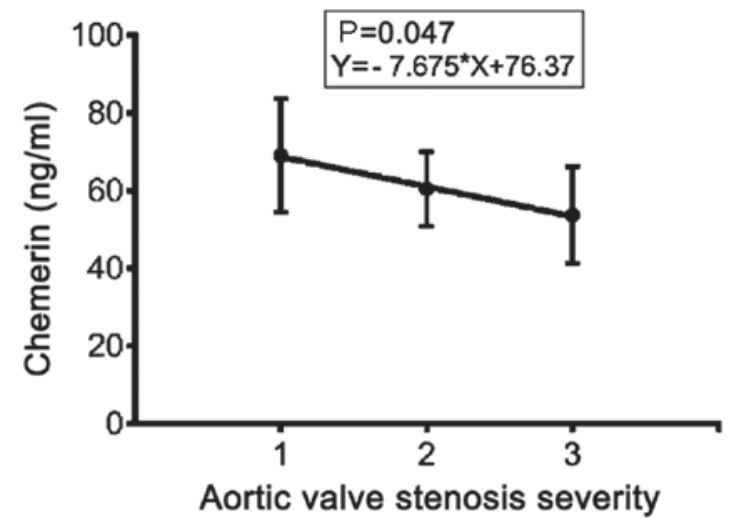

Figure 4. Regression line of the serum chemerin level in connection with the AoV stenosis severity grade.

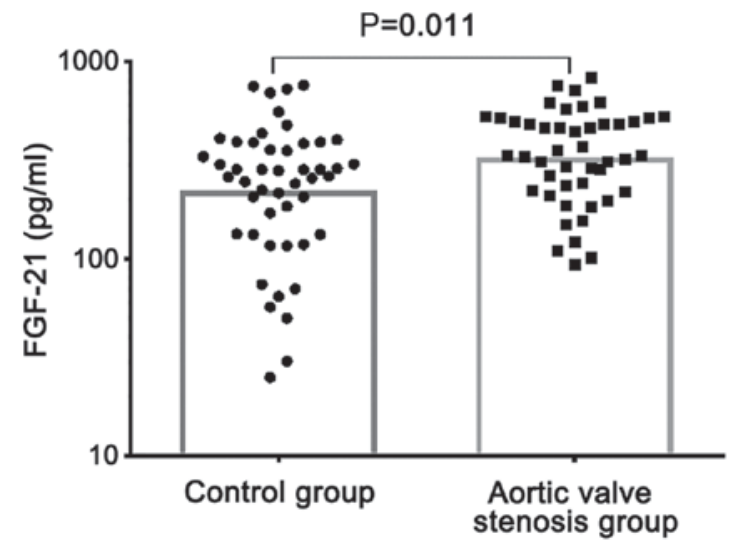

Figure 5. Serum FGF-21 level in the control and AoV stenosis group.

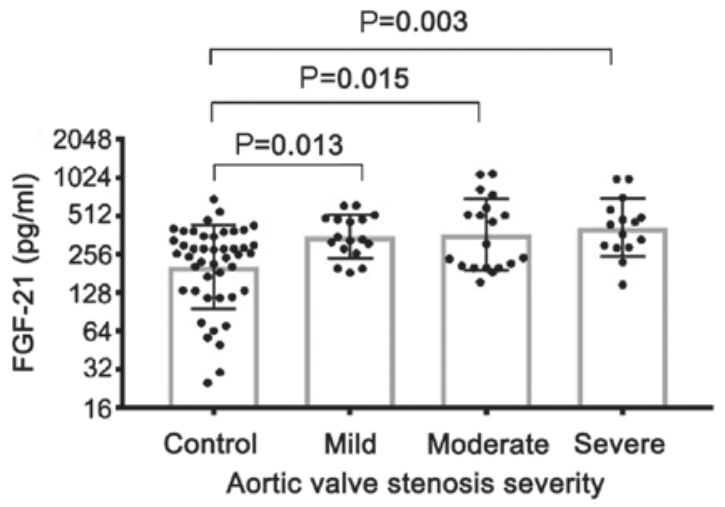

Figure 6. Serum FGF-21 expression level in the control group and in aortic valve stenosis groups with different grades of severity. FGF-21, fibroblast growth factor- 21 .

were divided into two groups (including control subjects). In order to determine more precisely the differences between the control and stenosis groups, distribution analysis was performed (Fig. 9B) and it was demonstrated that the distribution of MMP-1 was trimodal in the aortic stenosis group and monomodal in the control group. A total of $80 \%$ of patients in the aortic stenosis group (marked with a green oval) exhibited a significantly increased MMP-1 expression level compared with the control group ( $\mathrm{P}=0.0001$; Fig. 9C); however, MMP-1 


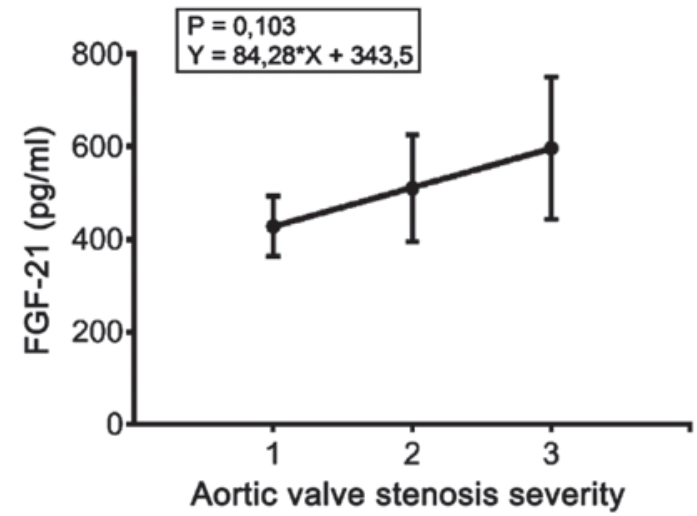

Figure 7. Regression line of the serum FGF-21 level and the aortic valve stenosis severity grade. FGF-21, fibroblast growth factor-21.

expression levels remained unaltered for $20 \%$ of patients with aortic stenosis results compared with the control group. The highest MMP-1 expression level was observed in the moderate AoV stenosis group $(\mathrm{P}<0.0001)$ and the lowest level in the severe aortic stenosis group $(\mathrm{P}=0.012)$, compared with the control patients (Fig. 9D). Analyses of serum expression levels of MMP-3, MMP-9, TIMP-1 and TIMP-3 between patients with aortic valve stenosis and the control patients, did not reveal any statistically significant differences (Fig. 10). A positive correlation was identified between MMP-1 and MMP-9 expression levels $\left(r_{p}=0.37\right.$; $P=0.017$; Fig. $\left.11 \mathrm{~A}\right)$; however, no associations were revealed between the expression levels of MMP-1 and MMP-3 and TIMP-1 (Fig. 11B and C).

\section{Discussion}

The present study analyzed the role of MMP-1 in the development of acquired calcific AoV stenosis, and identified the highest serum MMP-1 level in the moderate AoV stenosis group. The results of previous studies investigating the association between MMP-1 an AoV stenosis, the results demonstrated increased MMP-1 levels in damaged aortic valves resulting in degradation of the extracellular matrix components, including interstitial collagen types I, II and III, destruction of collagen, osteoblast differentiation and calcification $(7,15,28)$.

Solache-Berrocal et al (28) studied the association between the MMP-1 $1 \mathrm{G}>2 \mathrm{G}$ polymorphism and parameters indicative of mineralization in aortic valves from valve replacement. The results obtained by Solache-Berrocal et al confirmed the association between the highest amounts of calcium in valves with individuals carrying the allele resulting in increased transcription and translation of MMP-1. The association between 1G>2G MMP-1 polymorphism and calcium content of the aortic valve suggests that the $1 \mathrm{G}$ allele may exhibit a protective effect against calcium deposits.

The current study analyzed the results of serum MMP-1 expression among patients with AoV stenosis divided into two subgroups. In the current study, $20 \%$ of patients with AoV stenosis did not exhibit increased serum MMP-1 levels compared with the control group. Further analysis of expression levels among these patients revealed that all patients exhibited mild AoV stenosis. We assume that this stratification of the results into two subgroups (especially in patients of mild and
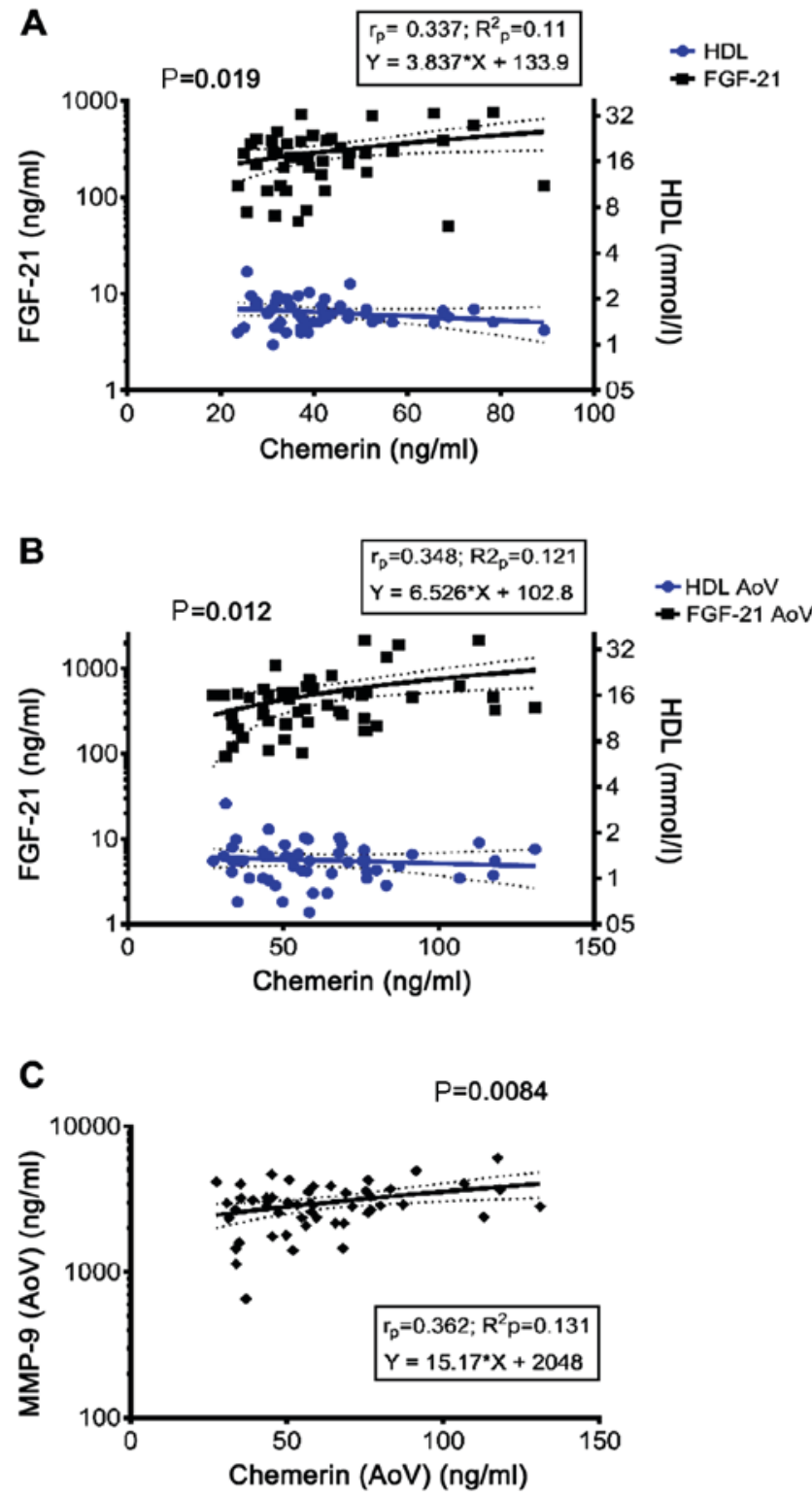

Figure 8. Regression analysis. Association analysis between serum chemerin and FGF-21, and chemerin and HDL (A) in the control and (B) in the AoV stenosis group. (C) Association between chemerin and MMP-9 in the AoV stenosis group. AoV, aortic valve; FGF-21, fibroblast growth factor-21; HDL, high-density lipoprotein.

moderate stenosis), may be required to determine whether $\mathrm{AoV}$ stenosis progressed more slowly among these patients. Other factors possibly involved in the pathogenesis of $\mathrm{AoV}$ stenosis should be considered. Mohty with co-authors (29) demonstrated that increased valvular oxLDL expression was associated with an increased tissue remodeling score, suggesting that oxLDL could be involved in the fibrocalcification of the aortic valve. The results of the current study and data from previous studies $(8,9,12,13,15,28)$ lead to a hypothesis that the most pronounced extracellular matrix remodeling occurs among patients with moderate AoV stenosis as the serum level of MMP-1 increases starting from the mild stenosis grade, reaches the maximum level in patients with moderate AoV stenosis, and decreases slightly in patients with severe AoV stenosis. Alterations in serum levels of MMP-1 observed in the present study in patients with different AoV stenosis severity grades 


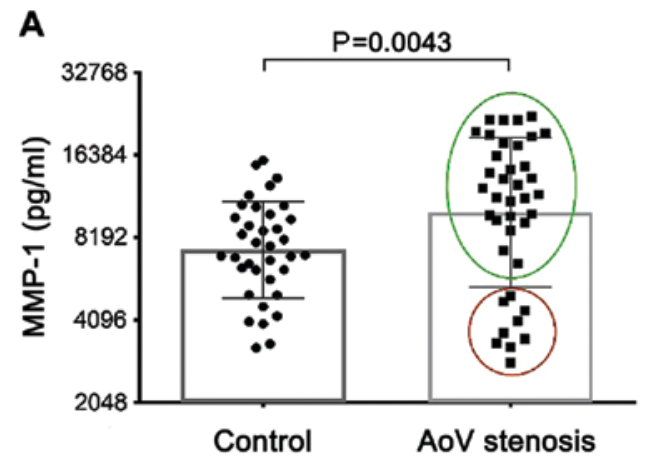

B

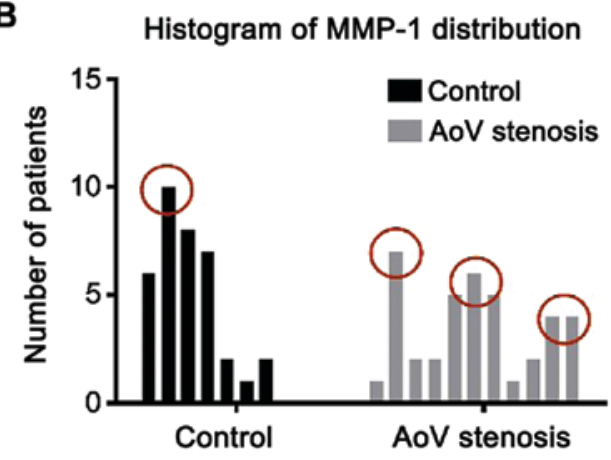

C

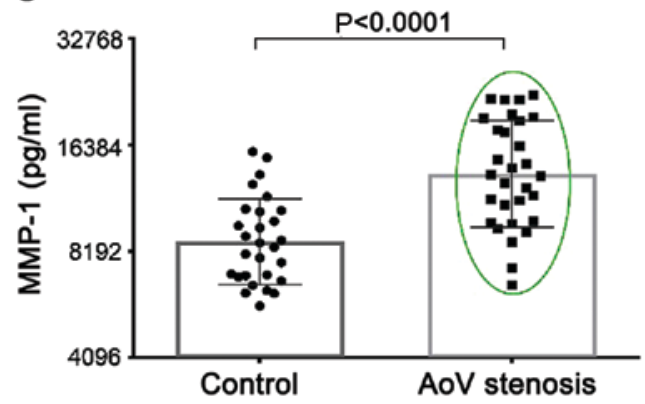

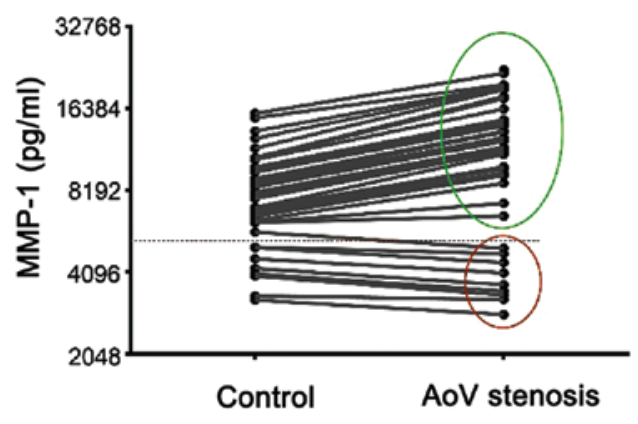

Histogram of MMP-1 distribution

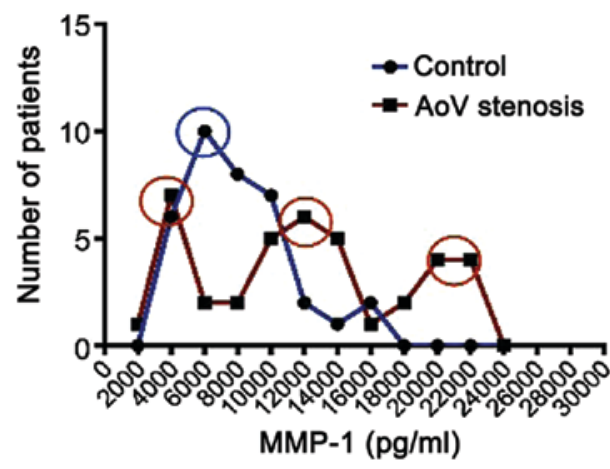

D

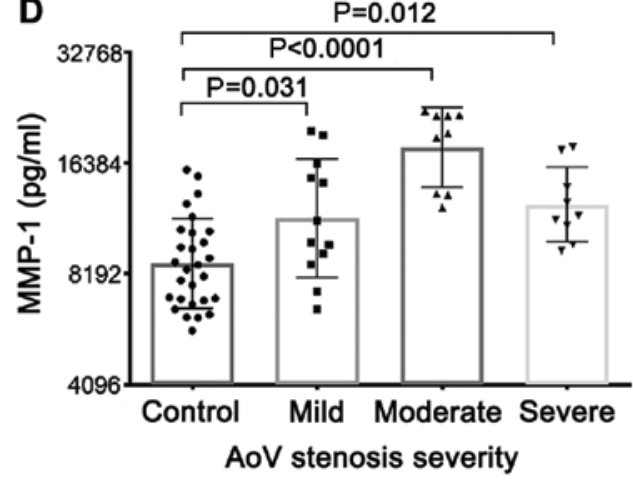

Figure 9. MMP-1 expression analysis. (A) Serum MMP-1 level in the control and AoV stenosis groups, ovals in green and red represent AoV stenosis patient clusters of data. (B) Histograms of MMP-1 distribution. Serum MMP-1 level. Modes are represented by color circles. (C) Serum MMP-1 levels in the control and AoV stenosis groups. Green ovals represent a cluster of $80 \%$ patients from (A) (D) MMP-1 serum level in control and in patients with different degrees of AoV severity. AoV, aortic valve; MMP-1, matrix metalloproteinase-1.

correspond with the results of other studies $(9,28)$ obtained using tissues and histological analyses. The lowest levels of MMP-1 were observed among patients with severe AoV stenosis, which may indicate low or absent inflammation at this stage of the disease and the development of valvular calcinosis.

In the present study, the serum expression level of TIMP-1 did not differ significantly between the control and AoV stenosis groups. Other authors have previously reported similar results. Kaden et al (9) studied the association between the inflammation in stenotic valves and the regulation of expression and activation of MMPs. It has been demonstrated that within lesions, leukocytes activated by the secretion of tumor necrosis factor- $\alpha$, may stimulate valvular myofibroblasts to proliferate and express MMP-1, while TIMP-1 remains unchanged (9). The expression of TIMP-1 was individually variable in both stenotic and control valves, showing no statistically significant difference between the groups.

The current study did not identify a correlation between the serum levels of TIMP-1 and MMP-1, which meant that TIMP-1 was excluded as a factor that causes an increase of MMP-1. Therefore, the upregulation of MMP-1 may serve as an explanation for the increased concentration of MMP-1 in patients with aortic stenosis, and this increase may be persistent and not regulated by TIMP. The analysis of serum levels of MMP-3 did not reveal a statistically significant difference between patients with AoV stenosis and the control group. Furthermore, the current study did not identify any significant differences between MMP-9 serum expression levels in patients with AoV stenosis and the control group patients. The above results indicate that the pathophysiological process in AoV stenosis is not the same 

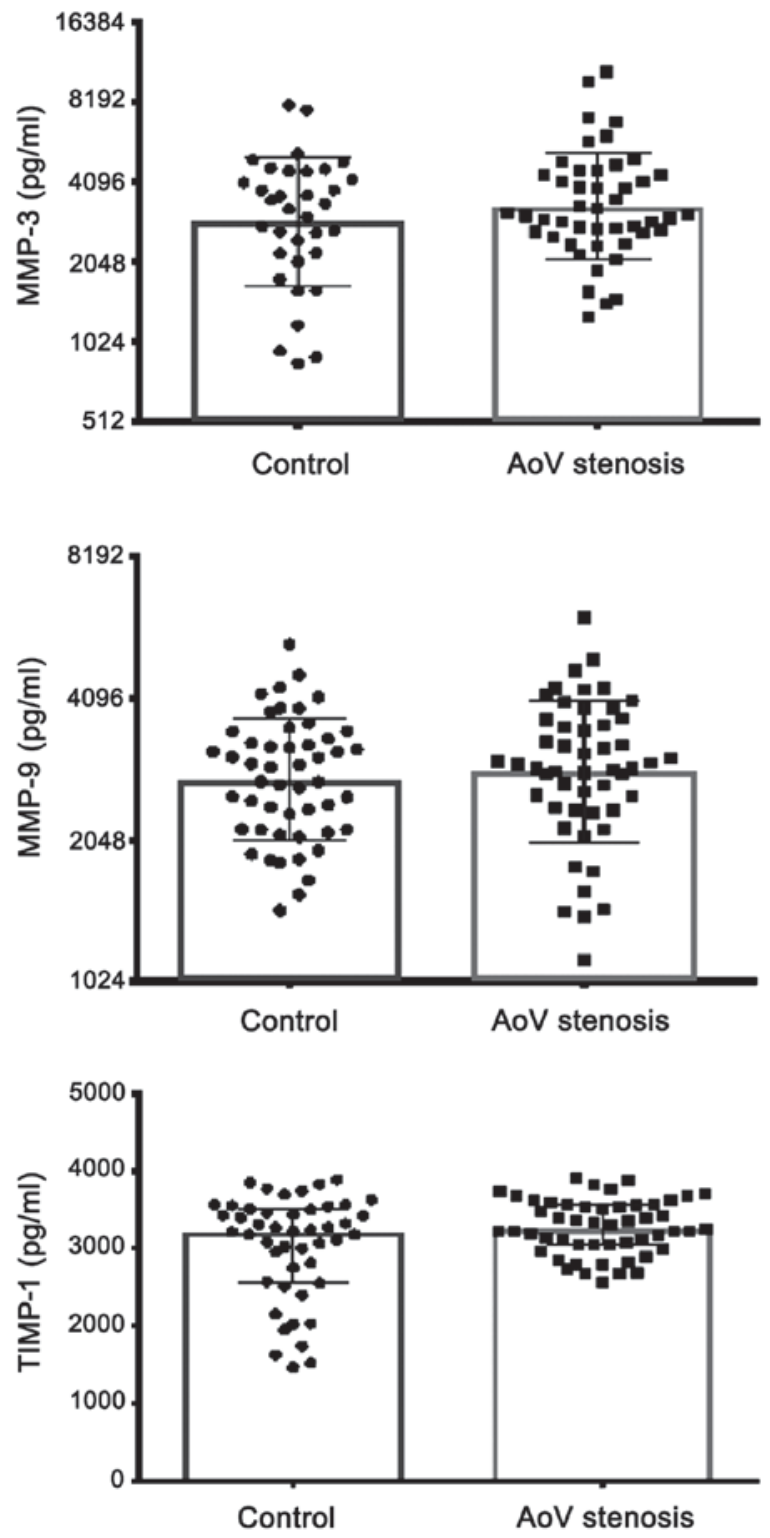

Figure 10. Serum levels of MMP-3, MMP-9, TIMP-1 in the control and AoV stenosis groups. MMP, matrix metalloproteinase; TIMP, tissue inhibitor of metalloproteinase; AoV, aortic valve.

as for atherosclerosis. Papazafiropoulou and Tentolouris (15) demonstrated that MMP-9 serves a central role in atherosclerosis, plaque formation, platelet aggregation, acute coronary syndrome, restenosis, aortic aneurysms and peripheral vascular disease. Increased MMP-9 levels are an independent risk factor of cardiovascular mortality among patients with coronary artery disease (16). The current study did not reveal an increase in MMP-9 serum levels in patients with aortic stenosis and did not identify an association between MMP-9 and TIMP-1 serum levels.

Satta et al (30) studied the expression of elastolytic MMPs and TIMPs in non-rheumatic aortic stenosis. The study concluded that the disproportion between MMP-9 and its tissue inhibitors may favor persistent MMP activation state within the calcific valve and contribute to the valvular remodeling process in the developing aortic stenosis. Satta et al also reported that MMP-9 protein was almost exclusively localized near or around the mineralized nodules, whereas the
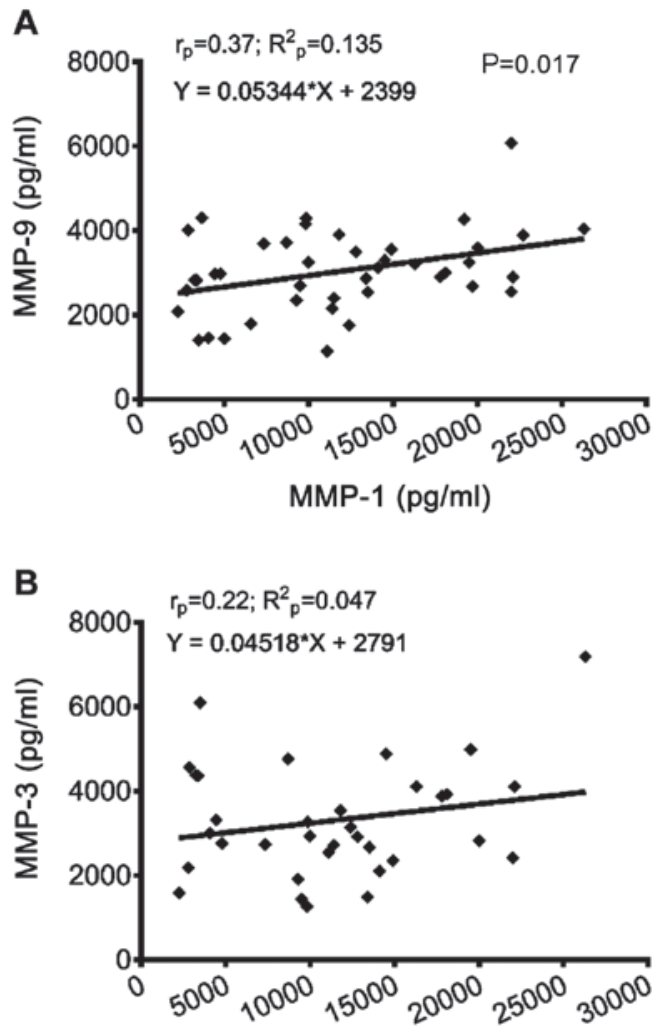

MMP-1 (pg/ml)

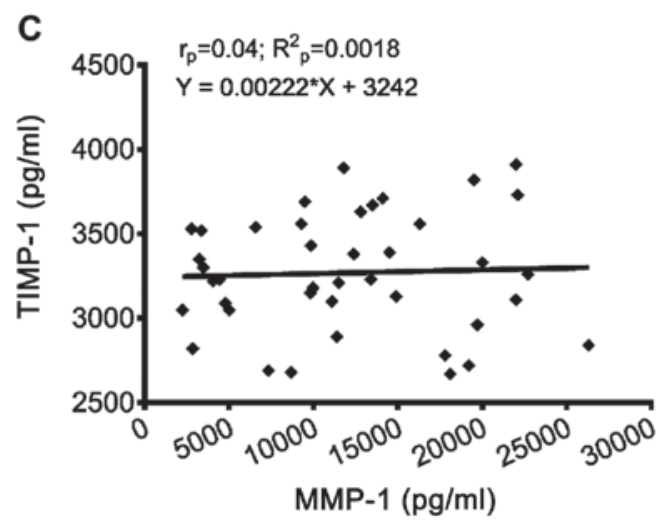

Figure 11. Regression analyses. Regression lines of serum (A) MMP-1 and MMP-9, (B) MMP-1 and MMP-3, and (C) MMP-1 and TIMP-1 in patients with AoV stenosis. AoV, aortic valve; MMP, matrix metalloproteinase.

expression was generally weak or absent in areas devoid of calcification.

The current study did not identify any correlation between the serum levels of TIMP-1 and MMP-9; however, a positive correlation was identified between the serum level of MMP-1 and MMP-9. The results of the current study were compared with those from previous studies involving histological material $(15,16,30)$ and based on these data, it was hypothesized that MMP-9 functions more locally at cellular level. The results of the current study indicated that MMP-1 may serve an important role in patients with aortic valve stenosis. Further studies on the role of MMPs in the development of AoV stenosis could result in clinical application of this knowledge.

The current study further analyzed the association between aortic valve stenosis and inflammatory factors including 
chemerin, C-reactive protein and FGF-21. Chemerin has been previously identified in different inflammatory and systemic processes $(31,32)$; and, therefore, patients with systemic and inflammatory diseases were excluded from the current study. Regn et al (17) demonstrated that pro-chemerin was expressed and secreted by the cardiac fibroblasts and converted to bioactive chemerin during proteolytic process, substantiating the idea that chemerin may exhibit both, pro- and anti-inflammatory functions. Analyzing the level of chemerin among patients with AoV stenosis and in the control group, the current study demonstrated that the expression chemerin was significantly increased among patients with AoV stenosis. Further analysis of AoV stenosis subgroups revealed that the highest serum chemerin level was present in the mild AoV stenosis group and a progressive decrease of chemerin levels was observed with an increasing degree of stenosis. In the control group the chemerin expression level was significantly lower compared with patients with severe AoV stenosis. Since chemerin is an inflammatory factor (28), its reduction with the progression of aortic stenosis suggests that the inflammation is prevalent at the beginning of the disease; however, calcinosis may predominate at later stages. The $\mathrm{C}$-reactive protein expression results further supported the hypothesis that at the beginning of AoV stenosis the inflammatory prices may be prevalent, as the expression levels of this protein were significantly increased in patients with mild stenosis compared with the control group. Galante et al (33) identified and described elevated C-reactive protein levels in degenerative aortic valve stenosis. The aforementioned results suggest that AoV stenosis at early stages may present as an active inflammatory process. At more severe stages, decreased serum chemerin levels suggest that extracellular matrix alterations and calcification may be the dominant processes in moderate and severe calcific AoV stenosis.

Chemerin has been studied as an inflammatory marker and FGF-21 as a factor that could induce a protective effect on the cardiac muscle in case of damage $(17,20)$. FGF-21 has been demonstrated to be released from the rat cardiac endothelial cells in vitro (18). FGF-21 was found to improve endothelial function, decrease oxidative stress and prevent endothelial cell apoptosis $(18,19,20,21)$. FGF-21 could serve as a biological marker of stress and damage to endothelial cells and may serve a physiological role in improving endothelial function (18). The expression of FGF-21 was significantly higher in patients with AoV stenosis compared with controls and further increased in patients with severe stenosis. The expression levels of serum chemerin were positively correlated with the levels of FGF-21. These results lead to a hypothesis that FGF-21 may serve a protective role in the pathogenesis of calcific AoV stenosis, as at the initial stages of the disease, only a slight increase in plasma FGF-21 was identified, while a greater increase was identified in patients with severe grade AoV stenosis. Previous studies further suggested that FGF-21 may exhibit a cardio protective role in different cardiovascular diseases including atrial fibrillation, myocardial infarction, coronary artery disease, hypertension, and cardiac hypertrophy $(19,20)$, supporting the results of the current study. FGF-21 also serves a role in the development of connective tissue (21). Considering the multiple effects FGF-21 and the association with connective tissue formation, it may be hypothesized that the increase in FGF-21 expression may be due to the progression of AoV stenosis and increased connective tissue formation.

The current data indicated that inflammation was dominant at the early, mild stage of AoV stenosis, the most pronounced extracellular matrix remodeling occurred in moderate AoV stenosis and calcification may have been the tominant process in severe calcific AoV stenosis. These results lead to a conclusion that aortic valve stenosis is an active inflammatory process which possibly could be delayed by therapeutic interventions.

To reduce the level of MMPs in patients with aortic valve stenosis, medications (including Nevibolol) that release nitric oxide (NO), could be prescribed, as NO inhibits MMPs production by endothelial cells and smooth muscle cells (15). Oxidized low density lipoprotein cholesterol has been observed to increase and high-density lipoprotein cholesterol to decrease the production of MMP-1 (34). This indicates that treatment of dyslipidemia remains necessary and may protect from the development of a more severe aortic stenosis. Since the elevated level of glucose induces the expression of MMP-1 and MMP-2 from endothelial cells and the expression of MMP-9 from macrophages, it is important to actively treat glucose metabolism disorders in order to ensure maximum possible normoglycemia (35). Based on the results of previous studies investigating the effects of drugs on MMP and TIMP levels, the adverse drugs include nitroglycerin (no long-acting nitrates have been studied, to the best of our knowledge), amlodipine and diltiazem, as well as ACE inhibitors, since angiotensin II decreases MMP-1 activity $(36,37)$. In cardiology, ACE inhibitors are a widely used drug group, and, therefore, the prevalence of progressive aortic stenosis could be partly explained by the wide use of this group of medicines.

\section{Acknowledgements}

Not applicable.

\section{Funding}

Not applicable.

\section{Availability of data and materials}

The datasets used and/or analyzed during the present study are available from the corresponding author on reasonable request.

\section{Authors' contributions}

JL and DL designed the study, analyzed and summarized the literature and were responsible for writing the manuscript. SS analyzed the data. ZK performed the experiments. VM, PT, $\mathrm{AL}, \mathrm{VR}$ and $\mathrm{VB}$ assisted in providing constructive analysis and interpreted the data. All authors read and approve the final manuscript.

\section{Ethics approval and consent to participate}

The study protocol was approved by the Riga Stradins University Ethics Committee on Research on Humans (Riga, Latvia) and the study protocol conforms to the Ethical Guidelines of the 1975 Declaration of Helsinki. Written informed consent was obtained from each patient enrolled in the current study. 


\section{Patient consent for publication}

Written informed consent was obtained from each patient enrolled in the current study.

\section{Competing interests}

The authors declare that they have no competing interests.

\section{References}

1. Beckmann E, Grau JB, Sainger R, Poggio P and Ferrari G: Insights into the use of biomarkers in calcific aortic valve disease. J Heart Valve Dis 19: 441-452, 2010.

2. Eveborn GW, Schirmer H, Heggelund G, Lunde P and Rasmussen K: The evolving epidemiology of valvular aortic stenosis. The troms $\emptyset$ study. Heart 99: 396-400, 2013.

3. Stewart BF, Siscovick D, Lind BK, Gardin JM, Gottdiener JS, Smith VE, Kitzman DW and Otto CM: Clinical factors associated with calcific aortic valve disease. Cardiovascular health study. J Am Coll Cardiol 29: 630-634, 1997.

4. Cosmi JE, Kort S, Tunick PA, Rosenzweig BP, Freedberg RS, Katz ES, Applebaum RM and Kronzon I: The risk of the development of aortic stenosis in patients with 'benign' aortic valve thickening. Arch Intern Med 162: 2345-2347, 2002.

5. Ren X: Aortic stenosis. https://emedicine.medscape.com/ article/150638-overview. Updated October 7, 2013. Accessed December 29, 2013.

6. Otto CM and Prendergast B: Aortic-valve stenosis-From patients at risk to severe valve obstruction. N Engl J Med 371: 744-756, 2014.

7. Lindman BR, Clavel MA, Mathieu P, Iung B, Lancellotti P, Otto CM and Pibarot P: Calcific aortic stenosis. Nat Rev Dis Primers 3: 16006, 2016.

8. Kaden JJ, Dempfle CE, Grobholz R, Tran HT, Kiliç R, Sarikoç A, Brueckmann M, Vahl C, Hagl S, Haase KK and Borggrefe M: Interleukin-1 beta promotes matrix metalloproteinase expression and cell proliferation in calcific aortic valve stenosis. Atherosclerosis 170: 205-211, 2003.

9. Kaden JJ, Dempfle CE, Grobholz R, Fischer CS, Vocke DC, Kiliç R, Sarikoç A, Piñol R, Hagl S, Lang S, et al: Inflammatory regulation of extracellular matrix remodeling in calcific aortic valve stenosis. Cardiovasc Pathol 14: 80-87, 2005.

10. Kaden JJ, Dempfle CE, Kiliç R, Sarikoç A, Hagl S, Lang S, Brueckmann $M$ and Borggrefe $M$ : Influence of receptor activator of nuclear factor kappa B on human aortic valve myofibroblasts. Exp Mol Pathol 78: 36-40, 2005.

11. Yetkin E and Waltenberger J: Molecular and cellular mechanisms of aortic stenosis. Int J Cardiol 135: 4-13, 2009.

12. Nagase $\mathrm{H}$ and Woessner JF Jr: Matrix metalloproteinases. J Biol Chem 274: 21491-21494, 1999.

13. Fondard O, Detaint D, Iung B, Choqueux C, Adle-Biassette H, Jarraya M, Hvass U, Couetil JP, Henin D, Michel JB, et al: Extracellular matrix remodelling in human aortic valve disease: The role of matrix metalloproteinases and their tissue inhibitors. Eur Heart J 26: 1333-1341, 2005

14. Heymans S, Schroen B, Vermeersch P, Milting $H$, Gao F, Kassner A, Gillijns H, Herijgers P, Flameng W, Carmeliet $\mathrm{P}$, et al: Increased cardiac expression of tissue inhibitor of metalloproteinase-1 and tissue inhibitor of metalloproteinase-2 is related to cardiac fibrosis and dysfunction in the chronic pressure-overloaded human heart. Circulation 112 1136-1144, 2005.

15. Papazafiropoulou A and Tentolouris N: Matrix metalloproteinases and cardiovascular diseases. Hippokratia 13: 76-82, 2009.

16. Blankenberg S, Rupprecht HJ, Poirier O, Bickel C, Smieja M, Hafner G, Meyer J, Cambien F, Tiret L and AtheroGene Investigators: Plasma concentrations and genetic variation of matrix metalloproteinase 9 and prognosis of patients with cardiovascular disease. Circulation 107: 1579-1585, 2003.

17. Regn M, Laggerbauer B, Jentzsch C, Ramanujam D, Ahles A, Sichler S, Calzada-Wack J, Koenen RR, Braun A, Nieswandt B and Engelhardt S: Peptidase inhibitor 16 is a membrane-tethered regulator of chemerin processing in the myocardium. J Mol Cell Cardiol 99: 57-64, 2016.
18. Domouzoglou EM, Naka KK, Vlahos AP, Papafaklis MI, Michalis LK, Tsatsoulis A and Maratos-Flier E: Fibroblast growth factors in cardiovascular disease: The emerging role of FGF21. Am J Physiol Heart Circ Physiol 309: H1029-H1038, 2015.

19. Han XY, Chen CY, Cheng G, Xie C, Yang M, Shou XL and Sun C: Serum fibroblast growth factor 21 levels are increased in atrial fibrillation patients. Cytokine 73: 176-180, 2015.

20. Planavila A, Redondo-Angulo I and Villarroya F: FGF21 and cardiac physiopathology. Front Endocrinol (Lausanne) 6: 133-139, 2015.

21. Schumacher JD and Guo GL: Regulation of hepatic stellate cells and fibrogenesis by fibroblast growth factors. Biomed Res Int 2016: 8323747, 2016

22. Vahanian A, Alfieri O, Andreotti F, Antunes MJ, BarónEsquivias G, Baumgartner H, Borger MA, Carrel TP, De Bonis M, et al: Guidelines on the management of valvular heart disease (version 2012): The joint task force on the management of valvular heart disease of the European society of cardiology (ESC) and the European association for cardio-thoracic surgery (EACTS). Eur Heart J 33: 2451-2496, 2012.

23. Lifshitz MS: Preanalysis. Blood collection overview. In: Henry's clinical diagnosis and management by laboratory methods, $23 \mathrm{e}$. McPherson RA and Pincus MR (eds.), Elsevier Inc., St. Louis, Missouri, pp24-26, 2017.

24. Trinder P: Determination of glucose in blood using glucose oxidase with an alternative oxygen acceptor. Ann Clin Biochem 6: 24-27, 1969

25. Izawa S, Okada M, Matsui $\mathrm{H}$ and Horita YJ: Quantitative determination of HDL cholesterol IVD. Med Pharm Sci 37: 1385-1388, 1997.

26. Benjamini Y, Krieger AM and Yekutieli D: Adaptive linear step-up procedures that control the false discovery rate. Biometrika 93: 491-507, 2006.

27. Lang RM, Bierig M, Devereux RB, Flachskampf FA, Foster E, Pellikka PA, Picard MH, Roman MJ, Seward J, Shanewise JS, et al: Recommendations for chamber quantification. J Am Soc Echocardiogr 18: 1440-1463, 2005.

28. Solache-Berrocal G, Barral A, Martín M, Román-García P, Llosa JC, Naves-Díaz M, Cannata-Andía JB and Rodríguez I: The association of MMP1 $1 \mathrm{G}>2 \mathrm{G}$ polymorphism with aortic valve. Rev Osteoporos Metab Miner 8: 115-120, 2016.

29. Mohty D, Pibarot P, Després JP, Côté C, Arsenault B, Cartier A, Cosnay P, Couture C and Mathieu P: Association between plasma LDL particle size, valvular accumulation of oxidized LDL, and inflammation in patients with aortic stenosis. Arterioscler Thromb Vasc Biol 28: 187-193, 2008.

30. Satta J, Oiva J, Salo T, Eriksen H, Ohtonen P, Biancari F, Juvonen TS and Soini Y: Evidence for an altered balance between matrix metalloproteinase-9 and its inhibitors in calcific aortic stenosis. Ann Thorac Surg 76: 681-688, 2003.

31. Rourke JL, Dranse HJ and Sinal CJ: Towards an integrative approach to understanding the role of chemerin in human health and disease. Obes Rev 14: 245-262, 2013.

32. Mariani F and Roncucci L: Chemerin/chemR23 axis in inflammation onset and resolution. Inflamm Res 64: 85-95, 2015.

33. Galante A, Pietroiusti A, Vellini M, Piccolo P, Possati G, De Bonis M, Grillo RL, Fontana C and Favalli C: C-reactive protein is increased in patients with degenerative aortic valvular stenosis. J Am Coll Cardiol 38: 1078-1082, 2001.

34. Ardans JA, Economou AP, Martinson JM Jr, Zhou M and Wahl LM: Oxidized low-density and high-density lipoproteins regulate the production of matrix metalloproteinase- 1 and -9 by activated monocytes. J Leukoc Biol 71: 1012-1018, 2002.

35. Death AK, Fisher EJ, McGrath KC and Yue DK: High glucose alters matrix metalloproteinase expression in two key vascular cells: Potential impact on atherosclerosis in diabetes. Atherosclerosis 168: 263-269, 2003

36. Eickelberg O, Roth M, Mussmann R, Rüdiger JJ, Tamm M, Perruchoud AP and Block LH: Calcium channel blockers activate the interleukin-6 gene via the transcription factors NF-IL6 and NF-kappaB in primary human vascular smooth muscle cells. Circulation 99: 2276-2282, 1999.

37. Funck RC, Wilke A, Rupp H and Brilla CG: Regulation and role of myocardial collagen matrix remodeling in hypertensive heart disease. Adv Exp Med Biol 432: 35-44, 1997.

This work is licensed under a Creative Commons

Attribution-NonCommercial-NoDerivatives 4.0 International (CC BY-NC-ND 4.0) License. 